\title{
Membrane Permeabilizing Activity of Pathogenesis-Related Protein Linusitin from Flax Seed
}

\author{
Sabina Anzlovar, ${ }^{1}$ Mauro Dalla Serra, ${ }^{2}$ Marina Dermastia, ${ }^{1}$ and Gianfranco Menestrina ${ }^{2}$ \\ ${ }^{1}$ Department of Biology, Biotechnical Faculty, University of Ljubljana, Vecna pot 111, 1000 Ljubljana, Slove- \\ nia; ${ }^{2}$ ITC-CNR Centro di Fisica degli Stati Aggregati, I-38050 Povo (TN), Italy \\ Accepted 4 March 1998.
}

\begin{abstract}
Linusitin is a 25-kDa pathogenesis-related (PR) protein of class 5 isolated from flax seeds. It has been proposed that PR-5 proteins exert their antifungal activity by permeabilizing fungal membranes. Using a fluorescent method that has been used for antimicrobial proteins other than PR proteins, we tested this hypothesis. The method is based on the release of the dye calcein from the inside of either small or large unilamellar lipid vesicles. The amount of calcein release induced by the protein was studied as a function of protein and lipid concentration and of membrane composition. All the results could be accounted for with an available statistical model. The model predicts that calcein release from the vesicles is a result of the tetrameric protein aggregation. Whether this aggregate corresponds to a transmembrane pore or to another protein complex that perturbs the membrane remains to be established. The lipid composition of the vesicles affected the permeabilization activity of linusitin. An increase of activity was observed by increasing the content of negatively charged phospholipids in the vesicles, inclusion of sterols into the membrane, or decreasing the $\mathrm{pH}$ of the solution. The possible roles of the observed changes in permeabilizing activity in actual plant-fungus interactions are discussed.
\end{abstract}

Additional keywords: Linum usitatissimum L., liposomes, thaumatin-like proteins.

The seed is highly protected in seed plants. The low water content and the seed coat provide effective physical barriers against bacterial and fungal invasion. However, during the imbibition phase preceding seed germination, these barriers gradually dissipate. Thereafter, protection relies mainly upon biochemical defenses, including the expression of different proteins (Bowles 1990), among them pathogenesis-related (PR) proteins (Stintzi et al. 1993). Based on serological properties, molecular mass, and sequence data, PR proteins have been grouped into five major classes (Van Loon et al. 1987).

The class 5 of PR proteins represents thaumatin-like proteins. They are acid-neutral or strongly basic proteins, located extracellularly, within the plant cell walls (Stintzi et al. 1993).

Corresponding author: Marina Dermastia; Fax: (386) 61273390

E-mail: marina.dermastia@uni-lj.si
Many of the proteins from this group have been reported to inhibit hyphal or spore growth of different fungi (Roberts and Selitrennikoff 1990; Woloshuk et al. 1991; Vigers et al. 1992; Huynh et al. 1992; Rodrigo et al. 1993; Vu and Huynh 1994; Koiwa et al. 1997; Hu and Reddy 1997). It has been proposed that the antifungal activity of these proteins is a result of permeabilization of fungal membranes (Roberts and Selitrennikoff 1990; Vigers et al. 1991).

The most-studied activity of the proteins from the PR-5 group is that of zeamatin isolated from maize (Roberts and Selitrennikoff 1990). Light microscopy and radiolabeling experiments have shown that in the presence of zeamatin, fungal hyphae leak and rupture just below their apices (Roberts and Selitrennikoff 1990). However, the mechanism of zeamatin action is not yet clear. Roberts and Selitrennikoff (1990) suggested that the molecule forms transmembrane pores, but the reported crystal structure of zeamatin (Batalia et al. 1996) is not thoroughly in accordance with this model. Therefore, Batalia et al. (1996) proposed that zeamatin action could be due to the electrostatic interaction with subapical hyphal structures, which in turn causes the influx of water and ions across the membrane.

Recently, a 25-kDa protein was isolated from flax seeds and its $\mathrm{N}$ terminus was sequenced (Borgmeyer et al. 1992). This newly isolated flax protein has $68 \%$ sequence identity to zeamatin, in addition to antifungal activity, and thus may have a similar, possibly permeabilizing, mechanism of action.

In our previous papers (Belmonte et al. 1993; Forti and Menestrina 1989; Menestrina 1988; Menestrina et al. 1989, 1991), we have studied the mode of action of several pore formers, or otherwise membrane-active compounds, by a fluorescent method based on the release of a dye, calcein, from the inside of lipid vesicles. In spite of the great potential and simplicity of this method, to our knowledge it has never been used for measuring PR-protein activity. In the present study, we used calcein release from lipid vesicles to test the hypothesis of membrane permeabilization by the $25-\mathrm{kDa}$ protein from flax seeds, and we derive the stoichometry of the permeabilizing unit.

\section{RESULTS AND DISCUSSION}

Biochemical characterization of 25-kDa protein.

We isolated a $25-\mathrm{kDa}$ protein from flax seeds by an extraction procedure modified from the one previously described by 
Borgmeyer et al. (1992). Using a single size exclusion chromatography column, instead of a cation-exchange column followed by reverse-phase chromatography (Borgmeyer et al. 1992), we were able to purify the protein in just one step (Fig. 1). The purity of the protein was confirmed by elution from a cation-exchange column (Fig. 1, inset). Accordingly, all the following experiments were performed with the active protein peak (Fig. 1) eluted from the size exclusion column.

The protein migrated, under reducing sodium dodecyl sulfate-polyacrylamide gel electrophoresis (SDS-PAGE), as a single band with a molecular mass of $25 \mathrm{kDa}$, and had an isoelectric point between 8.6 and 10 (Fig. 2).

For the antifungal assay (Roberts and Selitrennikoff 1986), $6 \mu \mathrm{g}$ of the purified $25-\mathrm{kDa}$ protein per disk inhibited growth of Alternaria alternata, which was scored as a crescentshaped retardation of the mycelial growth front (Anžlovar 1997).

Based on the antifungal activity of the $25-\mathrm{kDa}$ protein, the high degree of sequence homology of its first 37 amino acids with other class 5 PR proteins (Borgmeyer et al. 1992), and in accordance with accepted nomenclature (i.e.: thaumatin, Van der Wel and Loeve 1972; osmotin, Takeda et al. 1990; zeamatin, Roberts and Selitrennikoff 1990), we suggest that the 25-kDa protein from flax (Linum usitatissimum L.) be named linusitin.

\section{Permeabilization of lipid vesicles by linusitin.}

The permeabilizing activity of linusitin was tested on artificial membrane models. Lipid vesicles, either small unilamellar vesicles (SUVs) or large unilamellar vesicles (LUVs), were filled with the fluorescent dye calcein at a selfquenching concentration. The self-quenching effect was concentration dependent and started at calcein concentrations greater than $1 \mu \mathrm{M}$. Washed vesicles exposed to linusitin were monitored for the release of the entrapped dye. During permeabilization, calcein was released from the interior of the vesicles into the external solution. The dye became diluted by the external solution by several orders of magnitude. Therefore, the self-quenching effect disappeared and the total fluorescence of the sample increased with time. Such increase was proportional to the protein concentration and was expressed as the percentage of the calcein release (Fig. 3).

Because at a similar protein/lipid or protein/vesicle ratio LUVs were more permeabilized than SUVs, and since in LUVs phospholipids experience physico-chemical conditions more similar to that of cell plasma membranes, most of the following experiments were performed with LUVs.

The permeabilizing activity of an amount of linusitin that would normally induce $50 \%$ of calcein release from asolectin large vesicles was completely abolished by protein denaturation. Linusitin activity was destroyed by either extensive dithiothreitol reduction of disulfide bonds or by $15 \mathrm{~min}$ at $100^{\circ} \mathrm{C}$ (with or without dithiothreitol). This confirmed that permeabilization was due to a cysteine-rich protein and not to any nonprotein contaminant.

Effect of negatively charged lipids on the linusitin activity.

The lipid composition of the vesicles affected the permeabilizing activity of linusitin. Linusitin had little effect on neutral phosphatidylcholine vesicles, and it showed very little

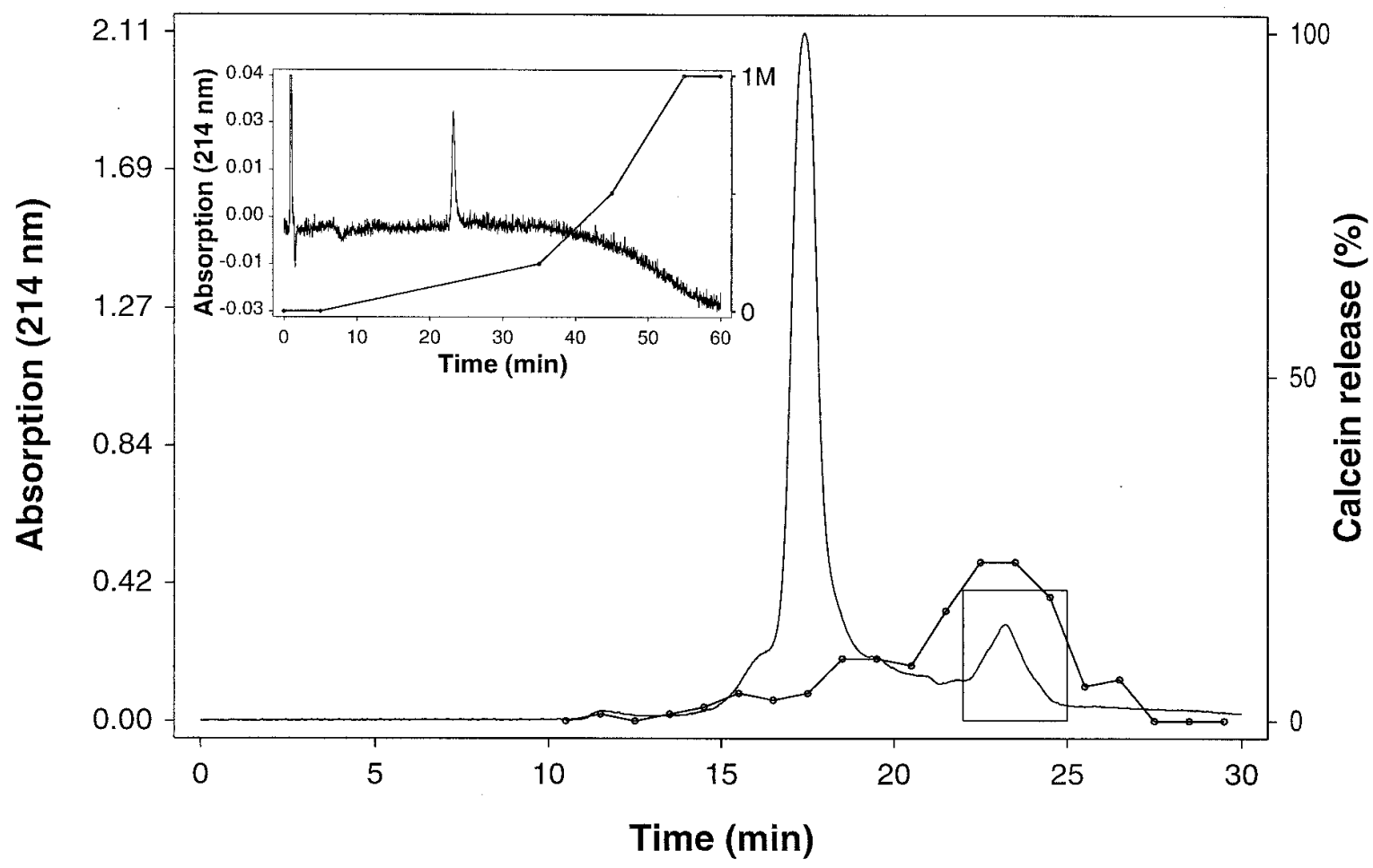

Fig. 1. Gel chromatography of flax seed crude extract by SEC 2000 (Beckman, Fullerton, CA) column (line without circles) and permeabilizing activity of fractions expressed as a percentage of calcein release from small unilamellar vesicles (line with circles). Inset: Ion exchange chromatography of the active peak from SEC 2000 column by MonoS HR 5/5 (Pharmacia, Uppsala, Sweden) column (line without circles). Gradient of NaCl from 0 to 1 M (line with circles). 
permeabilizing activity on phosphatidylcholine:phosphatidylserine vesicles composed in the molar ratio of 18:1 (Table 1). However, increasing the density of negative charges on the vesicles by lowering the ratio between phosphatidylcholine and negatively charged lipids (phosphatidylserine or phosphatidic acid) resulted in a more than 10-fold increase of calcein-releasing activity (Table 1). Permeabilization of vesicles composed of phosphatidylcholine and phosphatidylserine in a ratio $2.2: 1$ and 4.5:1 was significantly greater (20 and 14 times, respectively) than the control with LUV containing exclusively phosphatidylcholine (Table 1 ).

Although it is evident that the presence of a negative charge is significant in the protein-liposome interactions, it seems that the source of the negative charge is not as important. In fact, the permeabilizing activity was comparable when the vesicles were prepared with asolectin (Table 1), a mixture in which negatively charged phospholipids count for about $30 \%$ of the total lipids.

In addition, we tested linusitin-permeabilizing activity on human and bovine erythrocytes. The nonhemolytic response we observed (data not shown) agrees with previous results obtained with the vesicles of pure phosphatidylcholine. In fact, the periplasmatic layer of erythrocyte membrane is exclusively composed of electrically neutral lipids, mainly phosphatidylcholine and sphingomyelin and cholesterol, whereas acidic phospholipids, such as phosphatidylcholine, are in the inner layer (Op den Kamp 1979) and thus not freely accessible to linusitin.

\section{Effect of different sterols on linusitin activity.}

Considering the antifungal activity of linusitin (Borgmeyer et al. 1992) and the important role of membrane sterols in the vegetative growth and/or reproductive development of fungi (Weete 1989), we tested the hypothesis that inclusion of ster- ols in the target membrane might affect the permeabilizing activity of the PR-5 protein. We presumed that ergosterol, which is synthesized by the majority of higher fungi (Weete 1989) and represents $15 \%$ of their membrane lipids (Longley et al. 1986), might be a critical factor in the recognition process between plant and pathogenic fungus. Indeed, inclusion of sterols in vesicle membranes significantly increased the activity of linusitin, compared with the control without sterols. How-

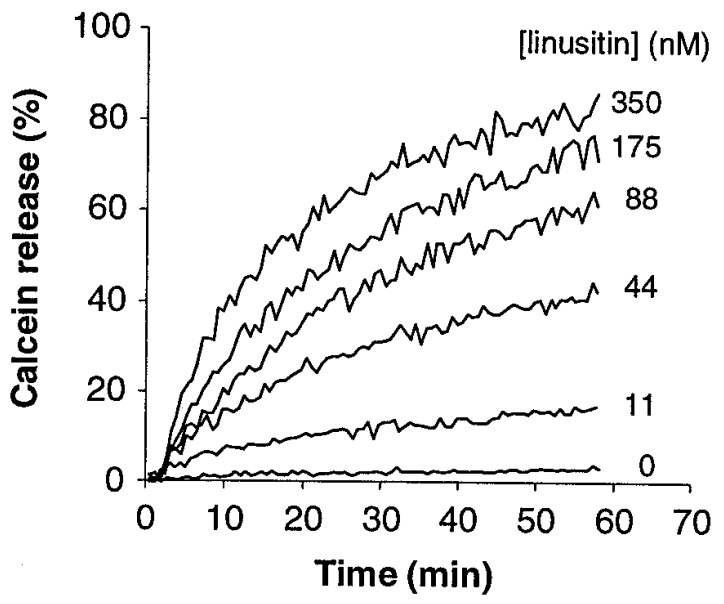

Fig. 3. Kinetics of linusitin-induced large unilamellar vesicle (LUV) permeabilization. Time course of fluorescence of calcein-loaded LUVs exposed to indicated amount of linusitin was measured with a fluorimeter. Percentage of calcein release was calculated as $\left(F(t)-F_{\text {fin }}\right) /\left(F_{\text {max }}-\right.$ $\left.F_{\text {in }}\right) \times 100$ where $F(t)$ is the fluorescence signal at time $t$. Other quantities are defined in Materials and Methods. Calcein release $(100 \%)$ was obtained with $1 \mathrm{mM}$ Triton X-100 (not shown). Lipid vesicles were composed of soybean asolectin at a constant concentration of $2.6 \mu \mathrm{M}$; external buffer was $20 \mathrm{mM}$ sodium acetate, $2 \mathrm{mM}$ EDTA, pH 5.0.
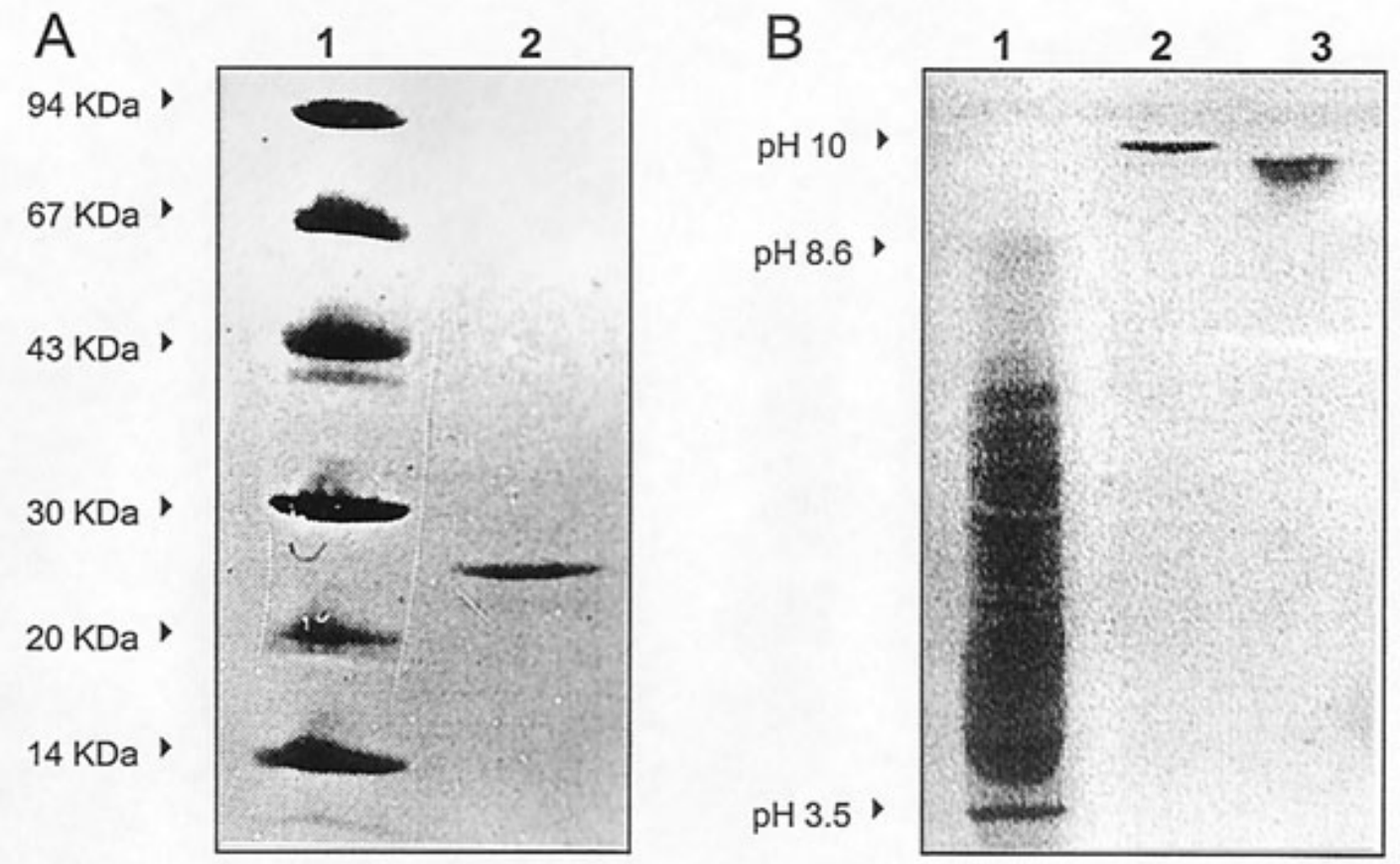

Fig. 2. (A) Sodium dodecyl sulfate-polyacrylamide gel electrophoresis and (B) isoelectric focusing of linusitin. Lanes A-1 and B-1, standards; lane B-2, EqT II, (Maček and Lebez 1988); lanes A-2 and B-3, purified linusitin. 
ever, cholesterol or stigmasterol enhanced the release of calcein up to 12 times compared with cholesterol-free vesicles, whereas ergosterol enhanced calcein release only 6 times (Table 2 ).

The influence of different sterols on the activities of various antimicrobial compounds has been previously demonstrated in yeast. A mutant of Saccharomyces cerevisiae lacking ergosterol was significantly less sensitive for binding of the phytotoxin syringomycin (Zhang and Takemoto 1986). To examine the hypothesis that membrane ergosterol is critical for syringomycin binding, Julmanop et al. (1993) studied the protective effect of externally added sterols on the cytotoxicity of syringomycin. The results were comparable to that on liposomes. Cholesterol had a pronounced effect on the cytotoxicity, stigmasterol was less effective, and ergosterol was the least effective. Furthermore, it has been suggested that membrane sterols are also involved in the antifungal activity of the peptidolipidic antibiotic iturin A. A mutant strain containing cholesterol instead of ergosterol was the most susceptible for iturin A (Latoud et al. 1990). Nevertheless, in the case of the antibacterial peptide magainin isolated from Xenopus skin that is also active against yeast, the incorporation of sterols in the liposomes reduced its lytic activity (Matsuzaki et al. 1995).

Various species of fungi have been reported to have different degrees of susceptibility to antifungal proteins, including PR-5 proteins (Stintzi et al. 1993; Vigers et al. 1992; Abad et al. 1996; Yun et al. 1997). As for the permeabilizing activity of linusitin, the presence of sterols in the membrane appears to be important and is consistent with the ability of the protein to stop the growth of different fungi. Interestingly, linusitin inhibited the growth of Alternaria solani (Borgmeyer et al. 1992) and Alternaria alternata (our experiment; Anžlovar 1997), but was ineffective against Phytophthora infestans (Borgmeyer et al. 1992), which does not synthesize any sterols (Weete 1989).

From our study, we conclude that neither sterols nor phospholipids in the fungal membrane represent a recognition site

Table 1. Effect of negative charge on the permeabilizing activity of linusitin $^{\mathrm{a}}$

\begin{tabular}{lcc}
\hline Lipid composition $^{\mathbf{b}}$ & Calcein release (\%) & \multicolumn{1}{c}{$\boldsymbol{S}^{\mathbf{d}}$} \\
\hline PC (control) & $3.5 \pm 3.5$ & \\
PC $:$ PS (18: 1) & $3.6 \pm 3.2$ & NS \\
PC $:$ PS (9:1) & $33.7 \pm 13.8$ & $<0.02$ \\
PC $:$ PS $(4.5: 1)$ & $50.1 \pm 7.6$ & $<0.002$ \\
PC $:$ PS $(2.2: 1)$ & $68.3 \pm 6.5$ & $<0.001$ \\
Asolectin & $58.0 \pm 11.8$ & $<0.005$ \\
PC $:$ PA (9:1) & 27.0 & \\
PC $:$ PA $(4.5: 1)$ & 45.0 & \\
\hline
\end{tabular}

${ }^{a}$ Linusitin $(132 \mathrm{nM})$ was added into the wells of a microtiter plate containing large unilamellar vesicles (LUVs) of different compositions in external buffer (20 mM Na-acetate, 2 mM EDTA, pH 5.0). Fluorescence was measured with a fluorimeter with interferometric filters at $485 \mathrm{~nm}$ for the excitation and at $538 \mathrm{~nm}$ for the emission. Results are expressed as a percentage of calcein release.

${ }^{b}$ In LUVs, ratios between neutral phosphatidylcholine (PC) and negatively charged phosphatidylserine (PS) or phosphatidic acid (PA) were decreased to obtain increasing concentrations of negative charges. Lipid concentration was $0.84 \mu \mathrm{M}$.

${ }^{c}$ Results are, except for PC : PA vesicles, the mean of four measurements $\pm \mathrm{SE}$

${ }^{\mathrm{d}}$ Calculation of the significance of differences between control PCvesicles and vesicles with increased negative charge was based on Student's $t$ test. NS = not significant. for linusitin. Yun et al. (1997) have proposed the existence of either a plasma membrane receptor for the PR-5 protein osmotin, or another facilitator of its activity. Although our model liposome system does not exclude this possibility, it shows that the optimal combination of membrane sterols and phospholipids is essential for the linusitin action.

\section{Dependence of the permeabilization on protein/lipid ratio and size of the permeabilizing unit.}

To obtain some insight into the mechanism of vesicle permeabilization by linusitin, we studied the dependence of calcein release on the concentration of reactants, i.e., protein and lipids. The experimental data were fitted with a theoretical model (described in Materials and Methods) that was derived from the one introduced by Rapaport et al. (1996). This model describes permeabilization as the result of the formation of an oligomeric protein lesion on the vesicles. Steady-state effects are explained in terms of three parameters: $M$, the minimal number of monomers necessary to form a lesion; $K_{1}$, the partition coefficient of the protein between water and lipid phase; and $K_{2}$, the equilibrium constant of the aggregation of proteins within the membrane, which is considered to be the same at each step during the formation of oligomers. Since we do not have independent evidence that calcein release is through a protein channel we currently cannot distinguish whether the lesion is a pore or a less defined perturbing unit, as discussed, for example, by Gazit et al. (1996).

Because the model does not have an exact analytical solution, it was solved by computer calculation, i.e., for every set of parameters the numerical solution of the model was computed as a function of lipid and protein concentrations. Therefore, to obtain a best fit, the three parameters of the model were varied independently over a very wide range of values and only those providing the best $\chi^{2}$ were selected for successive refinement. After these iterations, we found the triplet of values giving the best fit for each set of data. The level of confidence for the parameters was determined by comparing the predictions of the model when parameters were changed one at time around the best value (as exemplified in Figure 4 for parameter $M$ ). We observed that the formation of a tetrameric lesion is adequate to fit both LUV and SUV experiments. Furthermore, all our data could be described well with the same binding constant between protein and lipid, i.e., $K_{1}=(4$ $\pm 1) \cdot 10^{4} \mathrm{M}^{-1}$. A significant difference was observed in the

Table 2. Effect of sterols on permeabilizing activity of linusitin ${ }^{\mathrm{a}}$

\begin{tabular}{lcl}
\hline Lipid composition & Calcein release $(\boldsymbol{\%})^{\mathbf{b}}$ & \multicolumn{1}{c}{$\mathbf{S}^{\mathbf{c}}$} \\
\hline PC : PS (control) & $3.6 \pm 3.2$ & \\
PC : PS : ergosterol & $21.5 \pm 3.0$ & $<0.01$ \\
PC : PS : cholesterol & $42.0 \pm 3.5$ & $<0.0001$ \\
PC : PS : stigmasterol & $43.0 \pm 1.8$ & $<0.0001$ \\
\hline
\end{tabular}

${ }^{a}$ Linusitin $(132 \mathrm{nM})$ was added into the wells of a microtiter plate containing large unilamellar vesicles (LUVs) of different compositions in external buffer ( $20 \mathrm{mM}$ Na-acetate, $2 \mathrm{mM}$ EDTA, pH 5.0). Fluorescence was measured with a fluorimeter with interferometric filters at $485 \mathrm{~nm}$ for the excitation and at $538 \mathrm{~nm}$ for the emission. Results are expressed as a percentage of calcein release.

${ }^{\mathrm{b}}$ Results are the mean of four measurements \pm SE.

${ }^{\mathrm{c}}$ Calculation of the significance of differences between control PC : PS $=18: 1$ vesicles and vesicles composed of PC $:$ PS : ergosterol or cholesterol or stigmasterol (9:1:5 molar ratio) was based on Student's $t$ test. 
case of the aggregation constant $K_{2}$, which was $K_{2}=(3 \pm 1)$. $10^{-2}$ with LUV and $(4.5 \pm 1) \cdot 10^{-3}$ with SUV. This results in a lower tendency of the bound protein to aggregate on the surface of the SUV, with the overall effect that linusitin permeabilizes LUVs to a greater extent than SUVs.

This difference in linusitin aggregation might be a consequence of the packing of phospholipids in membranes with dissimilar curvatures (New 1990). In particular, as the curvature of the membrane increases in SUV, packing geometry restrictions dictate that over $70 \%$ of the lipids are in the outer
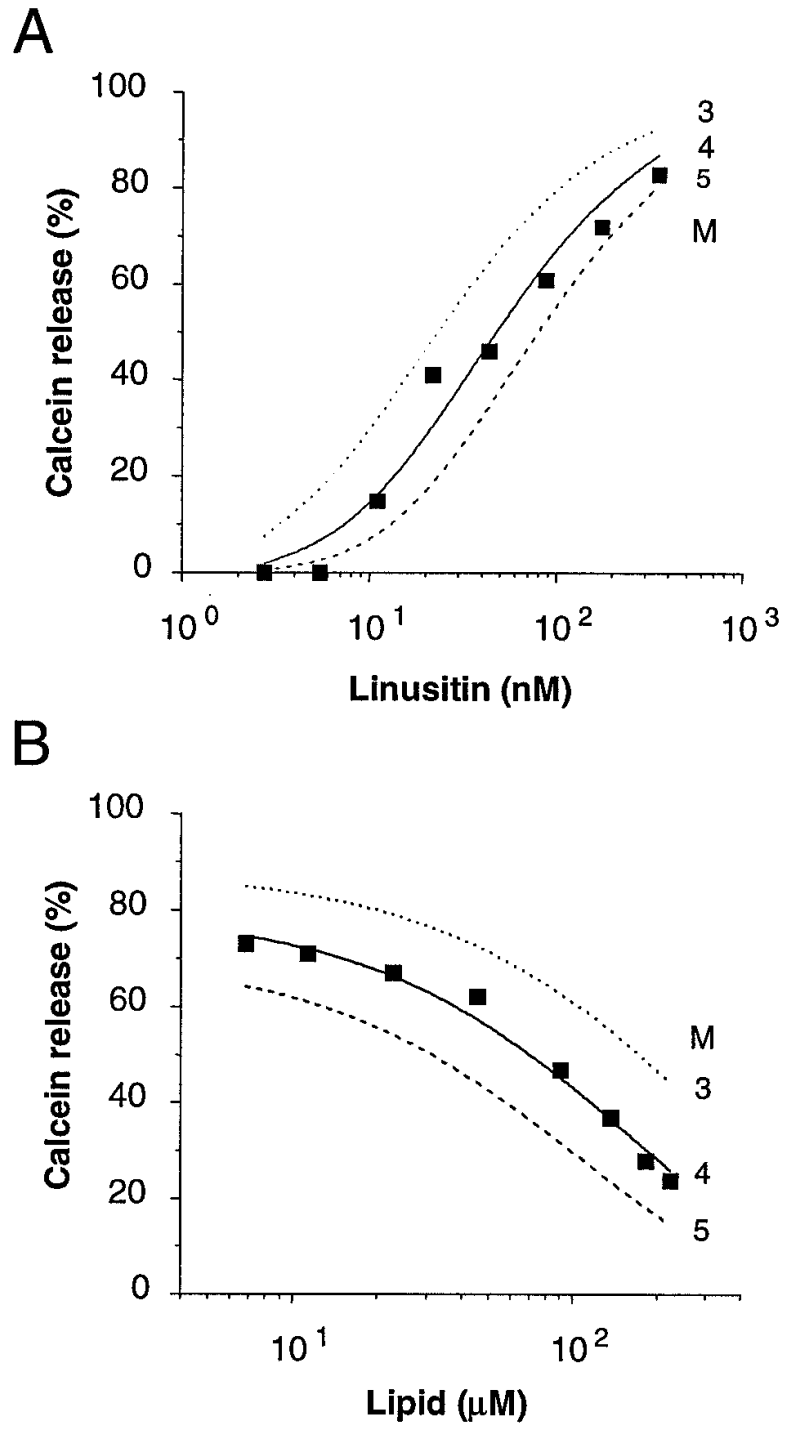

Fig. 4. Percentage of linusitin-induced, steady-state calcein release from large unilamellar vesicle (LUV) as a function of the protein or lipid concentration. Experiments at either (A) constant lipid or (B) constant protein concentration. Lipid vesicles were composed of soybean asolectin in a $20 \mathrm{mM}$ sodium acetate, $2 \mathrm{mM}$ EDTA, pH 5.0 buffer. Lines through the experimental points were calculated from the model and show how variations of the fitting parameters affected the predicted calcein leakage. In each case, two parameters $\left(K_{1}\right.$ and $\left.K_{2}\right)$ were kept constant at their optimal value, while the third $(M)$ was varied assuming the values reported. Best-fit values, corresponding to the solid lines, were as follows. For experiments at a constant lipid concentration of $2.6 \mu \mathrm{M}: M=4, K_{1}=$ $3.6 \cdot 10^{4} \mathrm{M}^{-1}, K_{2}=2.0 \cdot 10^{-2}$; for a constant protein concentration of $87.5 \mathrm{nM}: M=4, K_{1}=3.9 \cdot 10^{4} \mathrm{M}^{-1}, K_{2}=3.5 \cdot 10^{-2}$. leaflet. Vesicles composed of phospholipids with different headgroups also show asymmetry regarding the distribution of the lipids; those with smaller headgroups (e.g., phosphatidylserine and phosphatidic acid) preferentially occupy the inner layer, whereas phosphatidylcholine is found in the outer. This asymmetric packing could cause changes in linusitin aggregation rates both directly, depending on the geometry of the space occupied by the protein in the aggregated state, and indirectly, through the physical conditions of the outer layer (e.g., composition, lateral pressure, etc.).

pH and ionic strength dependence of linusitin activity.

Permeabilizing activity of linusitin increased with decreasing $\mathrm{pH}$ values ranging from 8.5 to 4.3 , with the highest activity at $\mathrm{pH} 4.3$ (Fig. 5A). Since the lipid vesicles were unstable at $\mathrm{pH}$ values lower than 4.0, we do not know if this is the real $\mathrm{pH}$ optimum. The total charge of membranes containing phosphatidylserine (but not phosphatidic acid or phosphatidylinositol) could change in this $\mathrm{pH}$ interval, becoming less negative (Cevc 1990). However, comparing the date shown in Table 1, this should decrease rather than increase linusitin activity. Alternatively, this basic protein could become even more positively charged at low $\mathrm{pH}$ values, at least in some

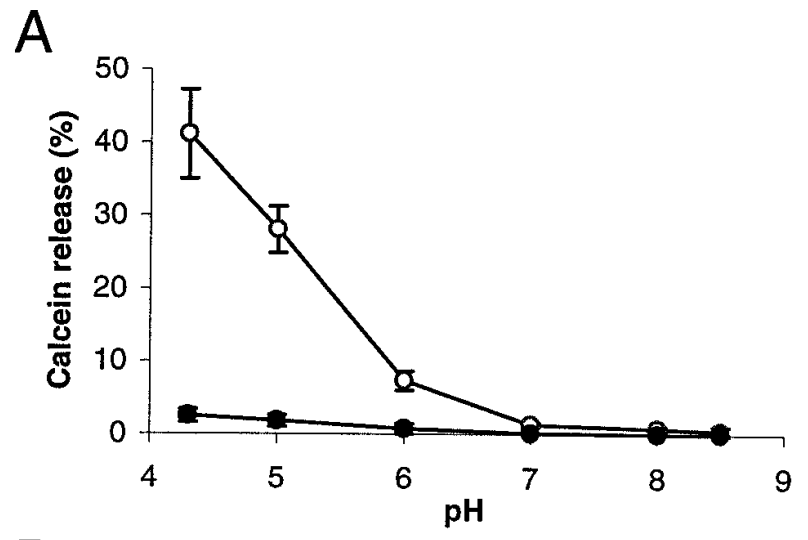

B

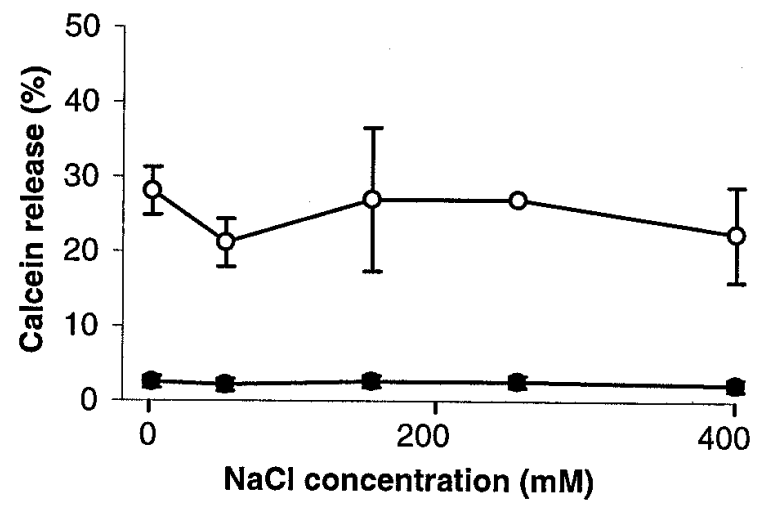

Fig. 5. Effect of (A) $\mathrm{pH}$ and (B) $\mathrm{NaCl}$ concentration on the permeabilizing activity of linusitin expressed as a percentage of calcein release. Linusitin (240 nM) (line with open circles) was added to the asolectin small unilamellar vesicles (SUVs) with a lipid concentration of $1.6 \mu \mathrm{M}$ in the external buffer ( $20 \mathrm{mM}$ Na-acetate, $2 \mathrm{mM}$ EDTA) with either varying $\mathrm{pH}$ values or a range of $\mathrm{NaCl}$ concentrations at a constant $\mathrm{pH}$ of 5. Controls (line with filled-in circles), containing everything except linusitin, show spontaneous leakage of calcein from the vesicles, in either set of experimental conditions. 
regions where acidic residues are exposed. This, in turn, could facilitate the interaction with the negative charges of the membrane via an electrostatic interaction. However, the fact that at $\mathrm{pH} 5.0$ the permeabilizing activity of linusitin is practically unaffected by the ionic strength (Fig. 5B) makes it less likely that electrostatic interaction is the reason for the higher activity at acidic $\mathrm{pH}$. It is possible that, as in the case of some bacterial toxins and pore-forming proteins such as diphtheria toxin, pseudomonal exotoxin A, and colicin (London 1992), low $\mathrm{pH}$ induces a new conformational state in the protein with increased affinity for the lipid phase.

In any case, the $\mathrm{pH}$ effect is so marked as to suggest that it might be functional for linusitin, implying that this protein may act in an acidic environment in vivo. Such conditions could be explained in the context of its potential role of defense during the seed germination process and in agreement with what is known for various other basic PR proteins (Stintzi et al. 1993), including its possible localization in the apoplast. Plant cells elongate irreversibly only when loadbearing bonds in the walls are cleaved. There is evidence from experiments with maize coleoptiles that wall loosening and growth are significantly acid induced, occurring only below pH 5 (Kutschera 1994). Regardless of the paucity of data available concerning cell-wall acidification during growth of plant organs other than stem and coleoptile sections (Rayle and Cleland 1992), the obtained $\mathrm{pH}$ range (Fig. 5A) suggests that linusitin defense activity in vivo could occur in an acidic apoplast, associated with the growth of the seedling.

\section{MATERIALS AND METHODS}

\section{Plant material.}

Seeds of flax (Linum usitatissimum L.) were obtained from Semenarna, Ljubljana, Slovenia.

\section{Isolation of the $25-\mathrm{kDa}$ protein.}

Extraction of the protein was performed exactly as described by Borgmeyer et al. (1992). The crude extract was filtered through a low protein binding filter, $0.22 \mu \mathrm{m}$ (Gelman, Ann Arbor, MI) and concentrated with an Ultrafree-CL filter, 5000 NMWL, regenerated cellulose membrane (Millipore, Bedford, MA). Fifty microliters of the sample was applied to a high-performance liquid chromatography gel filtration column (SEC 2000, Beckman, Fullerton, CA) equilibrated with $20 \mathrm{mM}$ sodium acetate buffer, $\mathrm{pH}$ 5.0. All fractions were collected and assayed for permeabilizing activity on liposomes.

A fraction that showed permeabilizing activity was concentrated (Ultrafree-CL filter, 5000 NMWL, regenerated cellulose membrane, Millipore) and applied to a MonoS cationexchange column equilibrated with $20 \mathrm{mM}$ sodium acetate buffer, pH 5.0 (mobile phase A). The elution was performed with mobile phase A over $5 \mathrm{~min}$, followed by a mixture of this with buffer $\mathrm{B}$ ( $20 \mathrm{mM}$ sodium acetate, $1 \mathrm{M} \mathrm{NaCl}, \mathrm{pH}$ 5.0) in which the concentration of $\mathrm{B}$ was gradually increased from 0 to $20 \%$ over $30 \mathrm{~min}$, from 20 to $50 \%$ over $10 \mathrm{~min}$, and finally from 50 to $100 \%$ over $10 \mathrm{~min}$.

\section{SDS-PAGE and isoelectric focusing.}

The purity of the protein was estimated by SDS-PAGE according to Laemmli (1970) with the PhastSystem (Pharmacia LKB Biotechnology AB, Uppsala, Sweden). The separating gel contained a gradient of acrylamide from 8 to $25 \%$ with $0.5 \%$ SDS. Gels were stained with Coomassie blue R-250 or with silver nitrate. Isoelectric focusing was performed in the same apparatus, with precast gels (Phastgel IEF, Pharmacia) with a $\mathrm{pH}$ gradient from 3 to 8.5 .

\section{Assay of antifungal activity.}

Antifungal activity against Alternaria alternata (Fr.:Fr.) Keissl. (ZIM L058, KPZL 101, Amica, Svica, 1990, Katalog biokultur 1995) was carried out with a hyphal extensioninhibition assay as described by Roberts and Selitrennikoff (1986).

\section{Preparation of liposomes.}

For the preparation of vesicles the following lipids were used: egg phosphatidylcholine and phosphatidylserine (both from Calbiochem, La Jolla, CA), phosphatidic acid (Avanti Polar Lipids, Alabaster, AL), cholesterol (Fluka, Buchs, Switzerland), ergosterol, stigmasterol (both from Sigma, Milan, Italy), and soybean asolectin (repurified from a Sigma product), which is a negatively charged mixture of $24 \%$ phosphatidylcholine, 39\% phosphatidylethanolamine, 19\% phosphatidylcholine, $6 \%$ phosphatidic acid, $1 \%$ phosphatidylinositol, and $11 \%$ other lipids. All mixtures given in the text are expressed as a molar ratio.

LUVs were prepared by extrusion of a solution containing freeze-thawed, multilamellar liposomes (Hope et al. 1985) in a buffer containing $80 \mathrm{mM}$ calcein (Sigma), and adjusted with $\mathrm{NaOH}$ to $\mathrm{pH}$ 6.8. Initial lipid concentration was $2 \mathrm{mg} / \mathrm{ml}$. Extrusion was performed with a LiposoFast Basic unit (Avestin, Ottawa, Canada) equipped with two stacked Nuclepore polycarbonate filters bearing holes with an average diameter of $100 \mathrm{~nm}$. Thirty-one passages were performed.

SUVs were prepared by sonication as described previously by Forti and Menestrina (1989). Briefly, the dried lipid mixtures were evaporated under reduced pressure. Lipids were suspended in calcein as above, vortexed, and sonicated for 30 min at room temperature with a pulsed sonicator (Vibracell VC 500, Sonics \& Materials, Danbury, CT).

To remove untrapped calcein, both types of vesicles were centrifuged through minicolumns containing Sephadex G50medium (Pharmacia), with a buffer of $10 \mathrm{mM}$ sodium acetate, $100 \mathrm{mM} \mathrm{NaCl}, 1 \mathrm{mM}$ EDTA, pH 6.0. Vesicle size was determined by quasi elastic light scattering with ZetaSizer 3 (Malvern Instruments, Malvern, U.K.), (as in Tejuca 1996). We measured an average diameter of approximately $90 \mathrm{~nm}$ and approximately $36 \mathrm{~nm}$ for LUVs and SUVs, respectively.

\section{Calcein release-based measurement of permeabilizing activity.}

For permeabilizing activity determinations, we used the calcein release method described by Kayalar and Duzgunes (1986), in conjunction with a microplate reader (Tejuca et al. 1996). Aliquots of washed SUVs or LUVs were introduced either into a stirred plastic cuvette containing $2 \mathrm{ml}$ of buffer (20 mM sodium acetate, $2 \mathrm{mM}$ EDTA, pH 5.0) or into the wells of a microtiter plate with $200-\mu 1$ aliquots of the above buffer. Cuvettes and microplates were incubated at $25^{\circ} \mathrm{C}$. Fluorescence in cuvettes was measured with a Jasco FP-550 spectrofluorimeter (Japan Spectroscopic, Tokyo), with an excitation wavelength at $494 \mathrm{~nm}$ and emission at $520 \mathrm{~nm}$ and 5- 
$\mathrm{nm}$ slits. For microplates, a filter fluorimeter was used (Fluostar, SLT, Labinstruments, Groeding, Austria), with interferometric filters at $485 \mathrm{~nm}$ for the excitation and at $538 \mathrm{~nm}$ for the emission. Maximum $(100 \%)$ calcein release $\left(F_{\max }\right)$ occurred with the addition of $1 \mathrm{mM}$ Triton X-100. The extent of permeabilization, $R_{\%}$, was calculated as a percentage of the maximum release obtained with Triton X-100, as follows: $R_{\%}$ $=\left(F_{\text {fin }}-F_{\text {in }}\right) /\left(F_{\text {max }}-F_{\text {in }}\right) \times 100$, where $F_{\text {in }}$ and $F_{\text {fin }}$ represent the initial and final value of fluorescence before and after protein addition, respectively. Spontaneous release of calcein from the vesicles in the buffer without the permeabilizing agent (i.e., linusitin or Triton X-100) was slow and could be disregarded for the purposes of this study.

\section{Theoretical model.}

The statistical model employed for our data analysis was first introduced by Parente et al. (1990) and later improved by Rapaport et al. (1996). Although we basically follow the treatment of these authors, for the sake of clarity, we summarize here briefly the basic assumptions and equations of the model.

The process of permeabilization is divided into two consecutive steps: (i) The protein monomers bind and incorporate into the lipid bilayer. (ii) The membrane-inserted monomers begin to aggregate.

When an aggregate has reached a critical size, a conducting unit is formed and the leakage of calcein can occur. We further assume that once a lesion has been formed the vesicle releases all of its contents.

The mathematical model is well described by three parameters. The first parameter is $M$, the minimal number of monomers necessary to form a lesion. The second parameter is $K_{1}$, the equilibrium constant of the incorporation process (step 1, above), which plays a role in the partition coefficient of the protein between water and lipid phase and can be written as follows:

$$
K_{1}=\frac{P_{b}}{\left(P_{0}-P_{b}\right) \cdot L}
$$

where $P_{0}$ and $L$ are the total concentration of protein and lipid respectively and $P_{b}$ is the concentration of protein bound.

The third parameter is $K_{2}$, the equilibrium constant of the aggregation of proteins within the membrane. For computational reasons, it is assumed here that the aggregation process is independent of aggregation as follows:

$$
K_{2}=\frac{X_{i+j}}{X_{\mathrm{i}} \cdot X_{\mathrm{j}}}
$$

where $X n$ is the number of oligomers of size $n$ per vesicle.

It follows that the percentage of released calcein is given by the following:

$$
R_{\%}=100 \sum_{i=M}^{N} A_{i} Z\left(M, i, K_{2}\right)
$$

where $A_{i}$ is the fraction of vesicles with $i$ monomers bound, $N$ is the maximum number of peptides that can be bound to one vesicle, and $Z\left(M, i, K_{2}\right)$ is the probability that a vesicle containing $i$ bound monomers will also have an aggregate of order not smaller than $M$.

$A_{i}$ will follow a binomial distribution so that

$$
A_{i}=\left(\begin{array}{c}
N \\
i
\end{array}\right) T^{i}(1-T)^{N-i}
$$

where $T$ is the probability of occupancy of one site, which cor- responds to the ratio between the protein bound and the total number of sites and is defined by the following:

$$
T=\frac{P b}{N \cdot V}=\frac{K_{1}}{1+K_{1} \cdot L} P_{0} \frac{v}{N}
$$

in which $V$ is the concentration of vesicles and $v$ stands for the number of lipid molecules per vesicle.

Finally, as discussed in Rapaport et al. (1996), $Z$ can be expressed as $Z\left(M, i, K_{2}\right)=p^{M-1}(M-M p+p)$, where

$$
p=\frac{1}{K+\sqrt{K^{2}-1}} ; K=1+\frac{1}{4 i K_{2}}
$$

\section{ACKNOWLEDGMENTS}

We thank Earl W. Taliercio and Susan J. Carlson for critical reading of the manuscript. This work was financially supported by a special grant from the Provincia Autonoma di Trento (PAT, 1913/CONV/1458) and in part by a fellowship from the Ministry of Science and Technology of Slovenia to S. A.

\section{LITERATURE CITED}

Abad, L. R., D’Urzo, M. P., Liu, D., Narasimhan, M. L., Reuveni, M., Zhu, J. K., Niu, X., Singh, N. K., Hasegawa, P. M., and Bressan, R. A. 1996. Antifungal activity of tobacco osmotin has specificity and involves plasma membrane permeabilization. Plant Sci. 118:11-23.

Anžlovar, S. 1997. Permeabilizing activity of a 25-kDa protein from flax seeds (Linum usitatissimum L.) on liposome membranes. M.Sc. thesis. University of Ljubljana, Ljubljana, Slovenia.

Batalia, M. A., Monzingo, A. F. Ernst, S., Roberts, W., and Robertus, J. D. 1996. The crystal structure of the antifungal protein zeamatin, a member of the thaumatin-like, PR-5 protein family. Nature Struct. Biol. 3:19-23.

Belmonte, G., Pederzolli, C., Maček, P., and Menestrina, G. 1993. Pore formation by the sea anemone cytolysin equinatoxin II in red blood cells and model lipid membranes. J. Membrane Biol. 131:11-22.

Borgmeyer, J. R., Smith, C. E., and Huynh, Q. K. 1992. Isolation and characterization of a $25-\mathrm{kDa}$ antifungal protein from flax seeds. Biochem. Biophys. Res. Commun. 187:480-487.

Bowles, D. J. 1990. Defense-related proteins in higher plants. Annu. Rev. Biochem. 59:873-907.

Cevc, G. 1990. Membrane electrostatics. Biochim. Biophys. Acta 1031: 311-382.

Forti, S., and Menestrina, G. 1989. Staphylococcal alpha-toxin increases the permeability of lipid vesicles by a cholesterol and $\mathrm{pH}$ dependent assembly of oligomeric channels. Eur. J. Biochem. 181:767-773.

Gazit, E., Miller, I. R., Biggin, P. C., Sansom, M. S. P., and Shai, Y. 1996. Structure and orientation of the mammalian antibacterial peptide cecropin P1 within phospholipid membranes. J. Mol. Biol. 258: 860-870.

Hope, M. J., Bally, M. B., Webb, G., and Cullis, P. R. 1985. Production of large unilamellar vesicles by a rapid extrusion procedure. Characterization of size distribution, trapped volume and ability to maintain a membrane potential. Biochem. Biophys. Acta 812:55-65.

Hu, X., and Reddy, A. S. N. 1997. Cloning and expression of a PR5-like protein from arabidopsis: Inhibition of fungal growth by bacterially expressed protein. Plant Mol. Biol. 34:949-959.

Huynh, Q. K., Borgmeywe, J. R., and Zobel, J. F. 1992. Isolation and characterisation of a $22 \mathrm{kDa}$ protein with antifungal properties from maize seeds. Biochem. Biophys. Res. Commun. 187:1-15.

Julmanop, C., Takano, Y., Takemoto, J. Y., and Miyakawa, T. 1993. Protection by sterols against the cytotoxicity of syringomycin in the yeast Saccharomyces cerevisiae. J. Gen. Microbiol. 139:2323-2327.

Kayalar, C., and Duzgunes, N. 1986. Membrane action of colicin E1: Detection of the release of carboxyfluorescein and calcein from liposomes. Biochem. Biophys. Acta 869:51-56.

Koiwa, H., Kato, H., Nakatsu, T., Oda, J., Yamada, Y., and Sato, F. 1997. Purification and characterization of tobacco pathogenesis related protein PR-5d, an antifungal thaumatin-like protein. Plant Cell Physiol. 38:783-791.

Kutschera, U. 1994. The current status of the acid-growth hypothesis. 
New Phytol. 126:549-569.

Laemmli, U. K. 1970. Cleavage of structural proteins during the assembly of the head of bacteriophage T4. Nature 227:680-685.

Latoud, C., Peypoux, F., and Michel, G. 1990. Interaction of iturin A, a lipopeptide antibiotic, with Saccharomyces cerevisiae cells: Influence of the sterol membrane composition. Can. J. Microbiol. 36:384-389.

London, E. 1992. How bacterial toxins enter cells: The role of partial unfolding in membrane translocation. Mol. Microbiol. 6:3277-3282.

Longley, R. P., Rose, A. H., and Knights, B. A. 1986. Composition of the protoplast membrane from Saccharomyces cerevisiae. Biochem. J. 108:401-412.

Matsuzaki, K., Sugishita, K., Fujii, N., and Miyajima, K. 1995. Molecular basis for membrane selectivity of an antimicrobial peptide, magainine 2. Biochemistry 34:3423-3429.

Maček, P., and Lebez, D. 1988. Isolation and characterization of three lethal and hemolytic toxins from the sea anemone Actinia equina $\mathrm{L}$. Toxicon 26:441-451.

Menestrina, G. 1988. Escherichia coli hemolysin permeabilizes small unilamellar vesicles loaded with calcein by a single hit mechanism. FEBS Lett. 232:217-220.

Menestrina, G., Forti, S., and Gambale, F. 1989. Interaction of tetanus toxin with lipid vesicles. Effects of $\mathrm{pH}$, surface charge and transmembrane potential on the kinetics of channel formation. Biophys. J. 55:393-405.

Menestrina, G., Pederzolli, C., Forti, S., and Gambale, F. 1991. Lipid interaction of Pseudomonas aeroginosa exotoxin A: Acid triggered aggregation and permeabilization of lipid vesicles. Biophys. J. 60:1388-1400.

New, R. R. C., ed. 1990. Liposomes: A Practical Approach. IRL Press, Oxford.

Op den Kamp, J. A. F. 1979. Lipid asymmetry in membranes. Annu. Rev. Biochem. 48:47-71.

Parente, R. A., Nir, S., and Szoka, F. C., Jr. 1990. Mechanism of leakage of phospholipid vesicle contents induced by the peptide GALA. Biochemistry 29:8720-8728

Rapaport, D., Peled, R., Nir, S., and Shai, Y. 1996. Reversible surface aggregation in pore formation by pardaxin. Biophys. J. 70:2502-2512.

Rayle, D. L., and Cleland, R. E. 1992. The acid growth theory of auxininduced cell elongation is alive and well. Plant Physiol. 99:12711274.

Roberts, W. K., and Selitrennikoff, C. P. 1986. Isolation and partial characterization of two antifungal proteins from barley. Biochim. Biophys. Acta 880:161-170.

Roberts, W. K., and Selitrennikoff, C. P. 1990. Zeamatin, an antifungal protein from maize with membrane-permeabilizing activity. J. Gen.
Microbiol. 136:1771-1778

Rodrigo, I., Vera, P., Tornero, P., Hernandez-Yago, J., and Conejero, V. 1993. CDNA cloning of viroid-induced tomato pathogenesis-related protein P23. Plant Physiol. 102:939-945.

Stintzi, A., Heitz, T., Wiedemann-Merdinoglu, S., Kauffmann, S., Geoffroy, P., Legrand, M., and Fritig, B. 1993. Plant pathogenesis-related proteins and their role in defence against pathogens. Biochimie 75 : 687-706.

Takeda, S., Sato, F., and Yamadry, I. K. 1990. Characterization of polypeptides that accumulate in cultured Nicotiana tabacum cells. Plant Cell Physiol. 31:215-221.

Tejuca, M., Dalla Serra, M., Ferreras, M., Lanio, M. E., and Menestrina, G. 1996. The mechanism of membrane permeabilization by sticholysin I, a cytolysin isolated from the venom of the sea anemone Stichodactyla helianthus. Biochemistry 35:14947-14957.

Van der Wel, H., and Loeve, K. 1972. Isolation and characterization of Thaumatin 1 and 2, the sweet-tasting proteins from Thaumatococcus daniellii Benth. Eur. J. Biochem. 31:221-225.

Van Loon, L. C., Gerritsen, A. M., and Ritter, C. E. 1987. Identification, purification and characterization of pathogenesis-related proteins from virus-infected Samsun NN tobacco leaves. Plant Mol. Biol. 9:593-609.

Vigers, A. J., Roberts, W. K., and Selitrennikoff, C. P. 1991. A new family of plant antifungal proteins. Mol. Plant-Microbe Interact. 4:315323.

Vigers, A. J., Wiedemann, S., Roberts, W. K., Legrand, M., Selitrennikoff, C. P., and Fritig, B. 1992. Thaumatin-like pathogenesis-related proteins are antifungal. Plant Sci. 83:155-161.

Vu, L., and Huynh, Q. K. 1994. Isolation and characterization of a 27$\mathrm{kDa}$ antifungal protein from the fruits of Diospyros texana. Biochem. Biophys. Res. Commun. 202:666-672.

Weete, J. D. 1989. Structure and function of sterols in fungi. Adv. Lipid Res. 23:115-167.

Woloshuk, C. P., Meunlenhoff, J. S., Sela-Buurgale, M., Van der Elzen, P. J. M., and Cornelissen, B. J. C. 1991. Pathogen-induced proteins with inhibitory activity toward Phytophthora infestans. Plant Cell 3: 619-628.

Yun, D., Zhao, Y., Pardo, J. M., Narasimhan, M. L., Damsz, B., Lee, H., Abad, L. R., D’Urzo, M. P., Hasegawa, P. M., and Bressan, R. A. 1997. Stress proteins on the yeast cell surface determine resistance to osmotin, a plant antifungal protein. Proc. Natl. Acad. Sci. USA 94: $7082-7087$.

Zhang, L., and Takemoto, J. Y. 1986. Mechanism of action of the Pseudomonas syringae phytotoxin, syringomycin. Interaction with the plasma membrane of the wild type and respiratory deficient strains of Saccharomyces cerevisiae. Biochim. Biophys. Acta 861:201-204. 